Research Article

\title{
Disorder-Induced Superconductor-Insulator Transition
}

\author{
Zeleke Deressa $\mathbb{D}^{1}$ and P. Singh ${ }^{2}$ \\ ${ }^{1}$ Ambo University, Department of Physics, Ambo, P.O.Box 19, Ethiopia \\ ${ }^{2}$ Addis Ababa University, Department of Physics, Addis Ababa, P.O.Box 1176, Ethiopia \\ Correspondence should be addressed to Zeleke Deressa; zelekederessagerbi@gmail.com
}

Received 2 April 2019; Revised 9 March 2020; Accepted 25 April 2020; Published 15 July 2020

Academic Editor: Yuri Galperin

Copyright $\odot 2020$ Zeleke Deressa and P. Singh. This is an open access article distributed under the Creative Commons Attribution License, which permits unrestricted use, distribution, and reproduction in any medium, provided the original work is properly cited.

\begin{abstract}
In this paper, we report the results of our theoretical investigation on the interplay of superconductivity and disorder in twodimensional (2D) systems. The effect of disorder on superconductivity of 2D systems was found analytically using Green's function formalism. The results of our calculation revealed that disorder induced due to randomly distributed superconducting islands enhances decoherence of Cooper pairs and suppresses superconductivity. We have also determined the critical value of disorder at which the 2D system completely loses its superconducting properties. Below this critical value of disorder, the system acts as a superconductor, a system with zero electrical resistance. Above the critical value, it acts as an insulator, a system with infinite electric resistance. This is a fascinating result because a direct transition from the state of the infinite conductivity to the opposite extreme of infinite resistivity is unexpected in the theory of condensed matter physics.
\end{abstract}

\section{Introduction}

Superconductivity is a resistanceless state of matter first discovered in mercury by Onnes [1]. This paradoxical state of matter was described microscopically by Bardeen et al. [2] in 1957. For this microscopic description, Bardeen et al. won the 1972 Nobel Prize in physics. The beauty of this state of matter is its large potential for application, especially in electric power transmission, fast train transportation, MRI medications, and quantum computing technologies. With this large potential for application, experimentalists have been searching for the elusive room-temperature superconductivity. This resulted in the discovery of high-temperature ceramic compounds [3], compounds of iron pnictides [4], and most recently in metallic hydrides under high pressure [5]. The conventional microscopic theory (BCS theory) failed to explain the mechanism of superconductivity in these high-temperature materials.

The study of the effect of disorder on superconductivity began in the late 1930s with the work of Shalnikov [6] and revived again in the late 1950s with the works of Anderson [7] and Abrikosov and Gor'kov [8]. Anderson showed that disorder solely by itself can destroy superconductivity and lead to insulating behavior in materials if sufficiently strong. Abrikosov and Gor'kov also showed that magnetic impurities of arbitrary concentration can destroy superconductivity of materials.

The problem of dirty superconductors gives a unique opportunity to study the competition between superconductivity which results from pairing of electrons and localization which results from scattering effects of nonmagnetic and magnetic impurities [7, 8], pair-breaking effects of disorderinduced Coulomb repulsion, or disorder-induced decoherence effects [9-13]. The interest in the field was further increased by the possibility that the disorder-driven or magnetic field-driven suppression of superconductivity in the limit of zero temperature might be a quantum phase transition [10].

Investigations in this field also revealed that the pairbreaking and decoherence effects of disorder on superconductivity of materials depend on their physical dimension and superconducting pairing symmetry. According to Anderson, weak nonmagnetic disorders (impurities, dislocations, etc.) which could not affect the time-reversal symmetry have no significant effect on thermodynamic properties of three-dimensional (3D) s-wave superconductors. In the literature, this is well known by Anderson's 
theorem. Until the late 1970s, most of the theoretical investigations in this field had been acting according to this theorem. However, the scaling theory of localization developed in 1979 by Abrahams et al. [14] revolutionized the study of dirty superconductors. According to this theory, two-dimensional (2D) systems are supposed to exist in only one of the two states at zero temperature, superconductor or insulator. There is no room for the metallic state to appear at this temperature because all electrons are expected to localize by infinitesimal amount of disorder. Moreover, unlike the case of 3D, infinitesimal amount of disorder can destroy superconductivity of 2D systems. As a result, 2D superconductors serve as ideal systems to study the less explored problem, the competition between superconductivity and localization. A complete suppression of superconductivity by disorder has been observed experimentally in granular and amorphous films such as $\mathrm{Bi}, \mathrm{Pb}$, Sn, Josephson junction arrays, and ${ }^{4} \mathrm{He}$ [15-17].

In the present paper, we have studied the effect of disorder on superconductivity of $2 \mathrm{D}$ systems. Based on the bosonic scenario of Mathew Fisher [18, 19], we have developed a Hamiltonian which describes our system and derived analytically the relationship between superconducting order parameter and disorder strength.

\section{Theoretical Model}

In two-dimensional superconductors, the effect of disorder can be either pair breaking or decoherence [20]. The pairbreaking effect is known by the fermionic model [21], and the decoherence effect is known by the bosonic model [19]. For disordered 2D systems, the most accepted scenario is the bosonic model. According to this model, superconducting islands of different sizes will be induced, and these randomsized islands can in turn induce random potentials which can potentially disturb the coherence between Cooper pairs of different islands. At some disorder strength, the coherence is completely lost, and the system becomes a gaped insulator. By the gaped insulator, we mean that there is a superconducting gap (also known as pseudo-gap) but no superconductivity. Figure 1, which was plotted by Bouadim et al. [21] based on quantum Monte Carlo simulation, depicts this model.

Based on this scenario, we developed a Hamiltonian of the form

$$
H=-t \sum_{i, v}\left(c_{i}^{+} c_{i+v}+c_{i} c_{i+v}^{+}\right)+U \sum_{i, v} n_{i} n_{i+v}-\sum_{i} \mu_{i} n_{i},
$$

where $c_{i}^{+}, c_{i}$, and $c_{i}$ represent the creation, annihilation, and number operators of Cooper pairs on the $i$-th lattice point, respectively. The subscript $v$ denotes the vector to the four nearest neighbours. $U$ and $t$ denote the repulsive interaction and the hopping integral for bosons on the nearest neighbour sites, respectively. $\mu_{i}$ represents the randomness in the single-site chemical potential due to variation in the island sizes. Deviation of the chemical potential $\Delta \mu_{i}$ from its average value $\mu, \Delta \mu_{i}=\mu_{i}-\mu$, is mainly due to distribution of the island size.

In the case of infinite on-site repulsive interaction, our bosonic model becomes a hard-core boson model, and double occupancy of bosons is completely restricted. Therefore, the hard-core boson system is a bosonic spin-half system. In order to transform bosonic Hamiltonian (1) into the Heisenberg-type spin Hamiltonian, we adopt the Matsubara-Matsuda transformation rule developed for hardcore bosons [22-25]:

$$
\begin{gathered}
c_{i}^{+} \longrightarrow S_{i}^{+}, \\
c_{i} \longrightarrow S_{i}^{-}, \\
c_{i}^{+} c_{i} \longrightarrow n-\frac{1}{2} .
\end{gathered}
$$

Employing this transformation rule, we have mapped our bosonic Hamiltonian into Heisenberg-type of the form

$$
H=-\frac{A_{x y}}{2} \sum_{i, v}\left(S_{i}^{+} S_{i+v}^{-}+S_{i}^{-} S_{i+v}^{+}\right)+A_{z} \sum_{i, v} S_{i}^{z} S_{i+v}^{z}-\sum_{i} \mu_{i} S_{i}^{z},
$$

where $A_{x y} \equiv t$ and $A_{z} \equiv U$ in equation (1). Starting from this spin-transformed Hamiltonian, we have calculated the averages in spin operators, $\left\langle S^{ \pm}\right\rangle$, employing Green's function formalism. In this model, these averages represent the superconducting order parameters. Therefore, based on the calculated averages, we can give a qualitative judgment whether the system is superconducting or insulating.

\section{Calculations}

In this section, we have calculated the expression for spin order which relates superconducting order parameter and disorder strength employing the equation of motion method of Green's function. The Fourier transformed equation of motion for retarded Green's function is

$$
\omega\left\langle\langle a, b\rangle_{\omega}=\langle[a, b]\rangle_{\omega}+\langle[a, H] ; b\rangle_{\omega} .\right.
$$

Replacing the Heisenberg operators $a$ and $b$ by spin operators $S_{i}^{+}$and $S_{j}^{-}$, respectively, substituting Hamiltonian (3) into the equation of motion (4) and computing the commutations on the RHS give

$$
\left\langle S_{k}^{+} ; S_{-k}^{-}\right\rangle_{\omega}=\frac{2\left\langle S^{z}\right\rangle}{\omega-4\left\langle S^{z}\right\rangle \omega_{k}-B},
$$

where

$$
\begin{gathered}
\omega_{k}=A_{z}-\frac{A_{x y}}{4} \sum_{v} e^{i \mathbf{k} \cdot v}, \\
B=\mu+\frac{\Delta \mu^{2}}{\mu} .
\end{gathered}
$$

In order to reduce the higher-order Green functions which appeared in our calculations, we have employed the following decoupling approximations:

$$
\begin{aligned}
& \left\langle\widehat{S}_{i}^{+} \widehat{S}_{i+v}^{z} ; \widehat{S}_{j}^{-}\right\rangle \equiv\left\langle\widehat{S}^{z}\right\rangle\left\langle\widehat{S}_{i}^{+} ; \widehat{S}_{j}^{-}\right\rangle, \\
& \left\langle\widehat{S}_{i}^{z} \widehat{S}_{i+v}^{+} ; \widehat{S}_{j}^{-}\right\rangle \equiv\left\langle\widehat{S}^{z}\right\rangle\left\langle\widehat{S}_{i+v}^{+} ; \widehat{S}_{j}^{-}\right\rangle .
\end{aligned}
$$




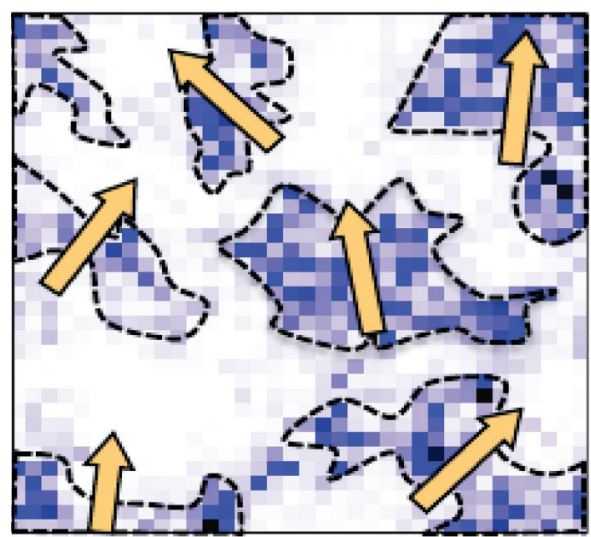

(a)

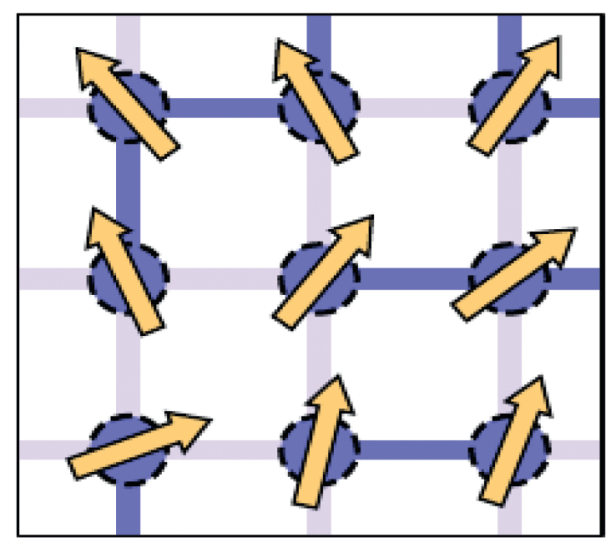

(b)

FIGURE 1: Superconducting islands separated by insulating regions (a). For very thin films, the islands are assumed to be on lattice sites (b) [21].

The spin operators were also transformed into $\mathbf{k}$-space representations as

$$
\begin{aligned}
\widehat{S}_{i}^{+} & =\frac{1}{\sqrt{N}} \sum_{k} e^{-i \mathbf{k} \cdot \mathbf{R}_{i}} \widehat{S}_{k}^{+}, \\
\widehat{S}_{i+v}^{+} & =\frac{1}{\sqrt{N}} \sum_{k} e^{-i \mathbf{k} \cdot\left(\mathbf{R}_{\mathbf{i}}+v\right)} \widehat{S}_{k}^{+}, \\
\widehat{S}_{j}^{-} & =\frac{1}{\sqrt{N}} \sum_{k} e^{-i \mathbf{k} \cdot \mathbf{R}_{\mathbf{j}} \widehat{S}_{-k}^{-} .}
\end{aligned}
$$

The correlation function for operators $a(t)$ and $b\left(t^{\prime}\right)$ is given by

$$
\left\langle b\left(t^{\prime}\right) a(t)\right\rangle=\int_{-\infty}^{\infty} \frac{S_{a b}(\omega)}{e^{\beta \omega}+1} e^{-i \omega\left(t-t^{\prime}\right)} \mathrm{d} \omega .
$$

The spectral density $S_{a b}(\omega)$ is given by

$$
S_{a b}(\omega)=\frac{i}{2 \pi}\left[G_{a b}^{\mathrm{ret}}\left(\omega+i 0^{+}\right)-G_{a b}^{\mathrm{adv}}\left(\omega-i 0^{+}\right)\right],
$$

where $G_{a b}^{\mathrm{ret}}(\omega)$ and $G_{a b}^{\mathrm{adv}}(\omega)$ are retarded and advanced Green's functions, respectively. Calculating the correlation function for the spin operators employing equation (9) and rearranging give

$$
\left\langle S^{x}\right\rangle \simeq\left(\frac{1}{4}-\frac{B^{2}}{16\left(A_{z}-A_{x y}\right)^{2}}\right)^{1 / 2} .
$$

Introducing normalized variables $\alpha \equiv \Delta \mu / A_{x y}$, $\beta \equiv A_{z} / A_{x y}$, and $\gamma \equiv \mu / A_{x y}$ and substituting into equation (11) finally give

$$
\left\langle S^{x}\right\rangle \simeq\left(\frac{16 \gamma^{2}(1-\beta)^{2}-\left(\gamma^{2}+\alpha^{2}\right)^{2}}{64 \gamma^{2}(1-\beta)^{2}}\right)^{1 / 2} .
$$

Equation (12) represents the interplay between superconducting order parameter and strengths of disorder and interaction between neighbouring Cooper pairs.

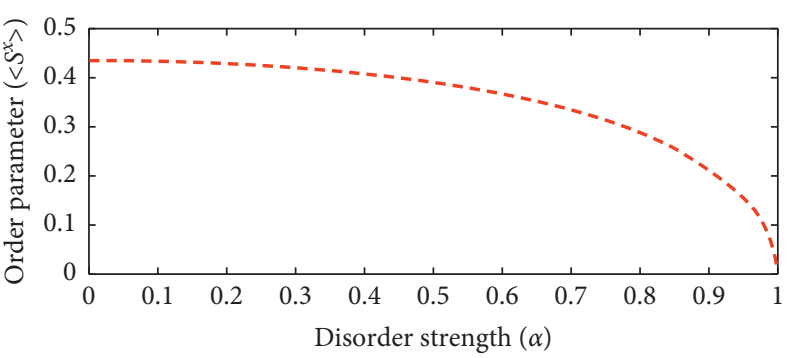

FIgURE 2: The behavior of superconducting order $\left\langle S^{x}\right\rangle$ with increasing disorder strength $\alpha$.

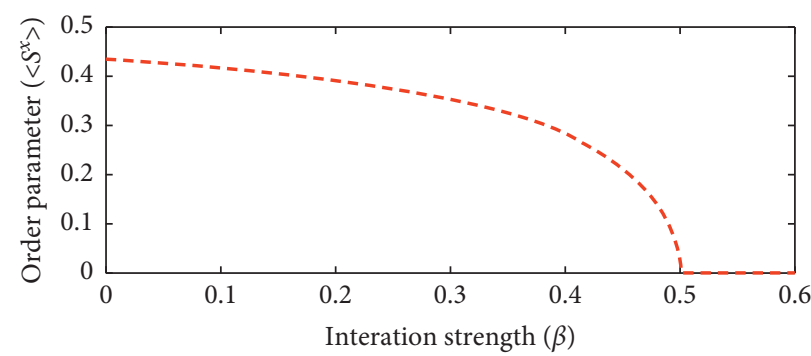

FIgURE 3: The behavior of superconducting order $\left\langle S^{x}\right\rangle$ with increasing interaction strength $\beta$.

\section{Results and Discussion}

The plot in Figure 2 indicates the interplay between disorder and superconductivity. As it has been clearly indicated, disorder suppresses superconductivity, and at some critical point, the system completely loses its superconductivity. Moreover, the strength of interaction advances the transition. Our theoretical result agrees with the experimental works of Goldman et al. [16, 17].

The plot in Figure 3 also depicts the interplay between superconductivity and strength of repulsive interaction between the neighbouring Cooper pairs. Just like disorder, the strength of repulsive interaction between Cooper pairs of neighbouring islands also suppresses superconductivity. At 
some critical value of the interaction strength, the system completely loses its superconductivity and becomes an insulator.

\section{Conclusion}

In this paper, we have studied the superconductor-insulator quantum phase transition in two-dimensional systems. We mainly considered the effect of disorder-enhanced randomness in an on-site chemical potential on the superconductivity of $2 \mathrm{D}$ thin films. To this end, we have calculated the relationship between the superconducting order parameter and strength of disorder in an on-site chemical potential. We have also calculated the relationship between superconducting order parameter and strength of repulsive interaction between Cooper pairs on neighbouring islands. Our calculation results revealed that disorder and repulsive Cooper pair interactions suppress superconductivity of $2 \mathrm{D}$ thin films. This result is very important in the study of strongly correlated electronic systems specifically in high-Tc copper-based superconductors. Here, we note that our calculations are based only on quantum mechanical meanfield approximations and can only explain qualitatively the more complex phenomenon of SIT. The quantitative explanation of this complex phenomenon requires to employ the more advanced quantum mechanical methods such as dynamical mean-field approximations and dynamical quantum Monte Carlo simulations [9, 21, 26, 27].

\section{Data Availability}

The manuscript is theoretical investigation. It does not have experimental/simulation data to avail for readers. The plots on the figures are generated from equations using Matlab. Therefore, all the data used to support the findings of this study are included within the article.

\section{Conflicts of Interest}

The authors declare that they have no conflicts of interest.

\section{References}

[1] H. K. Onnes, "Further experiments with liquid helium. C. On the change of electric resistance of pure metals at very low temperatures etc. IV. The resistance of pure mercury at helium temperatures," Koninklijke Nederlandse Akademie Van Wetenschappen Proceedings Series B Physical Sciences, vol. 13, no. 2, pp. 1274-1276, 1911.

[2] J. Bardeen, L. N. Cooper, and J. R. Schrieffer, "Microscopic theory of superconductivity," Physical Review, vol. 106, no. 1, pp. 162-164, 1957.

[3] J. G. Bednorz and K. A. Müller, "Possible high $T_{\mathrm{c}}$ superconductivity in the Ba-La-Cu-O system," Zeitschrift für Physik B Condensed Matter, vol. 64, no. 2, pp. 189-193, 1986.

[4] Y. Kamihara, T. Watanabe, M. Hirano, and H. Hosono, "Ironbased layered superconductor $\left.\mathrm{La}_{0} \mathrm{O}_{1-x} \mathrm{~F}_{x}\right] \mathrm{FeAs}(x=$ 0.05-0.12) with $T_{\mathrm{c}}=26 \mathrm{~K}$," Journal of the American Chemical Society, vol. 130, no. 11, pp. 3296-3297, 2008.

[5] I. Errea, M. Calandra, C. J. Pickard et al., "High-pressure hydrogen sulfide from first principles: a strongly an harmonic phonon-mediated superconductor," Physical Review Letters, vol. 114, no. 15, Article ID 157004, 2015.

[6] A. Shalnikov, "Superconducting thin films," Nature, vol. 142, no. 3584 , p. $74,1938$.

[7] P. W. Anderson, "Theory of dirty superconductors," Journal of Physics and Chemistry of Solids, vol. 11, no. 1-2, pp. 26-30, 1959.

[8] A. A. Abrikosov and L. P. Gor'kov, "Superconducting alloys at finite temperatures," Journal of Experimental and Theoretical Physics, vol. 38, no. 1, pp. 319-320, 1959.

[9] V. F. Gantmakher and V. T. Dolgopolov, "Superconductorinsulator quantum phase transition," Physics-Uspekhi, vol. 53, no. 1, pp. 1-49, 2010.

[10] V. Dobrosavljevic, N. Trivedi, and J. M. J. Valles, Conductor to Insulator Quantum Phase Transition, Oxford University Press, Oxford, UK, 2013.

[11] A. Ghosal, M. Randeria, and N. Trivedi, "Role of spatial amplitude fluctuations in highly disordereds-wave superconductors," Physical Review Letters, vol. 81, no. 18, pp. 3940-3943, 1998.

[12] R. T. Scalettar, N. Trivedi, and C. Huscroft, "Quantum Monte Carlo study of the disordered attractive Hubbard model," Physical Review B, vol. 59, no. 6, pp. 4364-4375, 1999.

[13] Y. Dubi, Y. Meir, and Y. Avishai, "Nature of the superconductor-insulator transition in disordered superconductors," Nature, vol. 449, no. 7164, pp. 876-880, 2007.

[14] E. Abrahams, P. W. Anderson, D. C. Licciardello, and T. V. Ramakrishnan, "Scaling theory of localization: absence of quantum diffusion in two dimensions," Physical Review Letters, vol. 42, no. 10, pp. 673-676, 1979.

[15] Y. Imry and M. Strongin, "Destruction of superconductivity in granular and highly disordered metals," Physical Review B, vol. 24 , no. 11 , pp. 6353-6360, 1981.

[16] A. M. Goldman and N. Markovic, "Superconductor-insulator transitions in the two-dimensional limit," Physics Today, vol. 51, no. 11, pp. 39-44, 1998.

[17] Y. Lin and A. M. Goldman, "Hard energy gap in the insulating regime of nominally granular films near the superconductorinsulator transition," Physical Review B, vol. 82, no. 21, Article ID 214511, 2010.

[18] M. P. A. Fisher, P. B. Weichman, G. Grinstein, and D. S. Fisher, "Boson localization and the superfluid-insulator transition," Physical Review B, vol. 40, no. 1, pp. 546-570, 1989.

[19] M. P. A. Fisher, "Quantum phase transitions in disordered two-dimensional superconductors," Physical Review Letters, vol. 65, no. 7, pp. 923-926, 1990.

[20] A. M. Finkelstein, "Superconducting transition temperature in amorphous films," JETP Letters, vol. 45, p. 46, 1987.

[21] K. Bouadim, Y. L. Loh, M. Randeria, and N. Trivedi, "Singleand two-particle energy gaps across the disorder-driven superconductor-insulator transition," Nature Physics, vol. 7, no. 11, pp. 884-889, 2011.

[22] T. Matsubara and H. Matsuda, "A lattice model of liquid helium," Progress of Theoretical Physics, vol. 16, no. 4, pp. 416-417, 1956.

[23] J.-X. Zhu, Inhomogeneous Superconductors, Oxford University Press, Oxford, UK, 1994.

[24] S. A. Owerre, "Magnon edge states in the hardcore- BoseHubbard model," Journal of Physics: Condensed Matter, vol. 28, no. 43, Article ID 436003, 2016.

[25] C. N. Varney, K. Sun, V. Galitski, and M. Rigol, "Quantum phases of hard-core bosons in a frustrated honeycomb lattice," New Journal of Physics, vol. 14, no. 11, Article ID 115028, 2012.

[26] M. V. Feigel'man, L. B. Ioffe, V. E. Kravtsov, and E. Cuevas, "Fractal superconductivity near localization threshold," Annals of Physics, vol. 325, pp. 1390-1478, 2010. 
[27] M. Stosiek, B. Lang, and F. Evers, "Self-consistent-field ensembles of disordered hamiltonians: efficient solver and application to superconducting films," 2019, http://arxiv.org/ abs/1903.10395. 